\title{
L' « Homo-Urgentus » dans les organisations : entre expression et confrontation de logiques d'urgence
}

Luc Bonneville et Sylvie Grosjean

\section{(2) OpenEdition}

12 Journals

Édition électronique

URL : http://journals.openedition.org/communicationorganisation/3367

DOI : 10.4000/communicationorganisation.3367

ISSN : 1775-3546

Éditeur

Presses universitaires de Bordeaux

\section{Édition imprimée}

Date de publication : 1 juin 2006

Pagination : 21-46

ISSN : 1168-5549

\section{Référence électronique}

Luc Bonneville et Sylvie Grosjean, «L' « Homo-Urgentus » dans les organisations : entre expression et confrontation de logiques d'urgence », Communication et organisation [En ligne], 29 | 2006, mis en ligne le 23 mai 2013, consulté le 19 décembre 2020. URL : http://journals.openedition.org/

communicationorganisation/3367 ; DOI : https://doi.org/10.4000/communicationorganisation.3367

Ce document a été généré automatiquement le 19 décembre 2020.

(C) Presses universitaires de Bordeaux 


\title{
L' « Homo-Urgentus » dans les organisations : entre expression et confrontation de logiques d'urgence
}

\author{
Luc Bonneville et Sylvie Grosjean
}

\section{Introduction}

Depuis environ deux décennies, les organisations, de plus en plus complexes, sont le théâtre d'une transformation qualitative de leur temporalité. Présentification, instantanéité, immédiateté, telles sont les épithètes qui caractérisent cette transformation, conformément au leitmotiv selon lequel il faut faire plus, le plus rapidement possible et avec moins. Les technologies de l'information et de la communication (TIC) participent à ce processus en permettant la mise à disposition et en circulation numériques de l'information ${ }^{1}$, ce qui constitue une véritable bifurcation dans l'histoire des techniques de communication ${ }^{2}$. Augmentation de la cadence de travail, intensification du travail, reengineering, ce sont là les quelques phénomènes auxquels sont confrontées les organisations soumises de plus en plus au joug d'un temps qui est désormais ramené à l'urgence ${ }^{3}$, laquelle s'impose maintenant non plus uniquement comme un moyen d'aller toujours plus vite pour atteindre telle ou telle chose, mais comme une finalité en soi réifiée en chose qui existe indépendamment des individus. C'est l'homo-urgentus qui désormais symbolise cette nouvelle façon de vivre, cette façon de se comporter en contexte organisationnel, là où la rapidité et la vitesse connotent au mépris de l'attente, des temps morts et de la lenteur.

Dans une première partie de ce texte, nous concentrerons notre attention sur l'évolution des représentations de la temporalité afin de souligner la montée du règne de l'urgence dans les sociétés contemporaines. Plus spécifiquement, nous insisterons sur ce qui caractérise le temps de la modernité à savoir la quantification et l'objectivité, attributs d'une société valorisant l'action et l'urgence comme modes privilégiés de régulation sociale, modes d'organisation de la vie collective ${ }^{4}$. Dans un deuxième temps, 
nous tenterons de souligner les enjeux sociaux, psychologiques, communicationnels, organisationnels et économiques de cette logique de l'urgence, notamment à partir d'un questionnement sur son mode d'expression et de confrontation au sein des organisations. Nous interrogerons notamment le rapport subjectif que l'homo-urgentus entretient avec le phénomène de l'urgence. Nous verrons que ce rapport a des modes d'expression différents d'un individu à un autre. Les uns manifesteront la volonté de faire plus et plus vite jouant ainsi le jeu de la «chrono-compétition $»^{5}$, tandis que les autres exprimeront des résistances en adoptant des "stratégies de freinage ». Par conséquent, deux logiques peuvent s'opposer et entrer en contradiction au sein de l'organisation ${ }^{6}$ : une logique de pouvoir et une logique de survie.

\section{Les représentations de la temporalité : du temps subjectif au temps objectif}

\section{Les transformations du rapport au temps}

3 La transformation du rapport au temps n'est pas un phénomène simple et observable sur une courte période ${ }^{7}$. C'est plutôt un phénomène lent, souvent très complexe, qui résulte de l'interaction de plusieurs phénomènes qui entrent en ligne de compte, souvent de façon dialectique, dans la transformation en question. Comme l'a montré Sue, le temps dans une société s'articule autour d'une représentation de la temporalité qui pose le temps comme objectif et global. De ce fait, le temps, d'un point de vue sociologique, est pour ainsi dire un fait social ${ }^{8}$ qui s'impose aux individus, en n'étant néanmoins jamais immuable mais toujours soumis aux tensions caractéristiques de la vie en société. Selon Sue ${ }^{9}$, ces tensions s'articulent autour d'un cycle perpétuel à la base de la représentation globale que les individus ont du temps.

Figure 1 : Le cycle historique des temps sociaux (Sue)

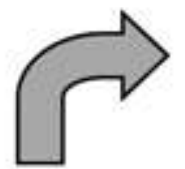

4. Formation d'un nouveau temps dominant

(Tension maximale, point de bascule)

\section{1. et 5 . Temps dominant}

(Monopole de la représentation temporelle)

\section{Affirmation de nouveaux}

temps sociaux

(Forme résiduelle)

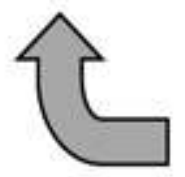

3. Déclin du temps dominant

(Contradiction montante avec

les autres temps sociaux)

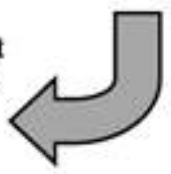

Ce modèle explicatif à perspective sociohistorique montre bien que le rapport au temps constitue l'une des voies d'analyses les plus pertinentes sur la façon dont les sociétés sont construites, en exprimant les différentes représentations que les êtres humains ont de la réalité qui les entoure. Les représentations de la temporalité sont à la fois le fondement et le résultat d'un temps tel qu'il se pose, ou tel qu'il est posé, à un moment 
donné de l'histoire, dans un espace donné. Avec l'espace, le temps est ainsi le cadre dans lequel se pose la réalité ${ }^{10}$. C'est d'abord ce temps qui mérite une attention particulière de la part des sciences sociales et humaines, en ce sens qu'il met en lumière le fondement même d'une société ${ }^{11}$. Ce temps, que nous nommerons "temps social», construit par des auteurs comme Mauss, Hubert, Halbwachs, etc. dont les travaux ont initialement été publiés dans la revue L'année sociologique, évoque l'idée selon laquelle toute temporalité est nécessairement le résultat d'une construction sociale. Le temps social exprime ainsi «[...] la nature et les rapports entre les divers modes d'activités dans le temps considérés selon leurs durées et leurs rythmes propres, de même que les différentes manières de concevoir et de se représenter le temps au sein de nos univers sociaux $» .^{12}$ De ce point de vue, ce n'est pas tant le temps en soi qu'il importe de comprendre mais plutôt la façon dont les individus, dans un lieu donné, à un moment donné, se le représentent ${ }^{13}$. C'est là l'une des clés, des dimensions centrales de la condition humaine ${ }^{14}$. Or, un grand nombre de travaux montrent que cette représentation de la temporalité a subi au cours de l'histoire plusieurs bouleversements significatifs ${ }^{15}$, lesquels mettent en lumière l'intime relation que le temps social entretient avec le changement social. D'où l'importance d'en faire ici l'étude, de façon on ne peut plus sommaire certes compte tenu de l'espace dont nous disposons, en ayant comme point de départ, inspirés en cela de Pronovost ${ }^{16}$, les distinctions centrales entre la représentation de la temporalité dans les sociétés pré-modernes (ou traditionnelles) et la représentation de la temporalité dans les sociétés modernes.

\section{La durée comme source première de la représentation de la temporalité}

5 À priori à toute construction sociale du temps, la représentation subjective de la temporalité, celle qui a pu être dominante dans les sociétés pré-modernes, s'articule autour d'un certain « rapport biologique » de l'être humain à la réalité qui l'entoure ${ }^{17}$. De ce rapport se construit une représentation intimement liée au corps comme objet de la nature, ou du moins comme objet lui étant soumis. Il s'agit là, essentiellement, d'un temps qui est, d'abord et avant tout, subjectivement vécu, faisant par là partie de l'expérience individuelle et singulière de chaque individu qui vit le temps à partir d'une intensité psychologique d'où découle une perception de la durée ${ }^{18}$. Rythmes respiratoires, cardiaques, digestifs, etc. constituent, en ce sens, autant de facteurs ayant une incidence sur la façon dont on perçoit le temps ${ }^{19}$. Mais cette représentation de la temporalité propre aux sociétés traditionnelles est beaucoup plus complexe puisqu'elle se structure aussi, et en très grande partie, par la relation que le corps entretient avec la nature proprement dite. Mentionnons ici le rôle déterminant des cycles solaires, lunaires, etc., qui façonnent la représentation du temps chez l'individu toujours en lien avec la conscience qu'il a des phénomènes qui l'entourent d'une part, et de la place de son propre corps dans la nature d'autre part ${ }^{20}$.

6 Sur une autre échelle, l'individu typique des sociétés traditionnelles, comme l'ont déjà montré plusieurs ${ }^{21}$, se représente la temporalité suivant les croyances auxquelles ils adhèrent. Croyances modelées par les valeurs, les rituels et le sacré qui vont déterminer, aussi, comment la société et les individus doivent organiser leur temps à partir d'une autorité souvent signifiée par un récit mythique ${ }^{22}$. Le temps sacré est ici considéré dans un rapport à la circularité, par une mémoire collective où l'on revit 
ensemble dans les rites ou les fêtes un moment originel qui marque une coupure entre un temps sacré et un temps profane ${ }^{23}$. Ces rythmes religieux se répètent séquentiellement, ponctuellement, pour révéler une temporalité cyclique où la fin est source du début et le début source de la fin. En ce sens, comme l'indique Sue, la notion linéaire du temps dans les sociétés pré-modernes n'existe pas ${ }^{24}$. Ce n'est qu'avec la modernité que la linéarité, objectivement quantifiée, s'impose dans toutes les sphères d'activités sociales.

\section{Le temps de la modernité : quantification et objectivité}

7 L'avènement de la modernité en Occident est marqué partout par l'essor de l'industrialisation, de la science et de la technique ${ }^{25}$. Dans un mouvement dialectique complexe d'interactions entre les individus et les nouvelles structures socioéconomiques qui se mettent alors en place, on voit apparaître et se généraliser dans l'ensemble des rapports sociaux une temporalité objective, quantifiable et quantifiée, qui débouche sur une nouvelle structuration des activités dans le temps ${ }^{26}$. Ce temps réussi à se substituer à la durée, au sens bergsonien du terme, telle que vécue par les individus. Ainsi cette durée perd-t-elle toute son importance au profit d'une "séquentiation " qui obéit au rythme des secondes, des minutes et des heures. De la naissance à la mort, l'être humain se trouve plongé dans un univers où règne une certaine logique "quantificatrice " par laquelle le temps devient une unité - donc acquiert une valeur définie par un nombre - qui s'accumule et qui chemine vers un "devenir " qui est celui de l'espérance à l'évolution sociale et à la prospérité économique, conforme à la notion de progrès de l'esprit humain tel qu'imaginé par Condorcet $^{27}$. C'est ce temps objectif, abstrait ${ }^{28}$, qui va se généraliser dans l'ensemble du tissu social par le recours systématique à certaines unités de mesure, telles que la clepsydre, le cadran, l'horloge, la montre, etc., qui vont conduire à une uniformisation de la durée, des séquences et des rythmes. Le temps repose ici sur une norme abstraite qui s'érige en système temporel dominant, celui du temps de travail lui-même modelé par les grandes structures économiques bourgeoises : «Peu à peu, la bourgeoisie s'installe au pouvoir et organise la vie des autres et la sienne propre en une chaîne continue d'événements datés: le temps pour le travail, le temps pour le repos, le temps pour la plaisir [...] $»{ }^{29}$ Dans cette nouvelle représentation de la temporalité, le temps devient l'instrument par excellence de la régulation des rapports économiques et, par extension, des rapports sociaux dans leur ensemble. Le travail devient central et avec lui le temps de travail autour duquel vont s'articuler l'ensemble des temps sociaux tels que le temps de repos, le temps de loisir, le temps pour la fête, etc. Le temps se conjugue maintenant avec des cadences, des rythmes et des horaires imposés: "La cadence, c'est la vitesse du mouvement, en tant qu'il est mesuré par la répétition des gestes ou des opérations dans une unité de temps donné. [...] Le rythme correspond à un agencement des cadences. Il se définit par la succession de cadences plus ou moins rapides, plus ou moins lentes, voire nulles, et éventuellement de temps de repos. La distribution des temps de travail à des cadences différentes et des temps morts compose un rythme. [...] L'horaire [...] délimite la durée du travail dans l'écoulement quotidien du temps. L'heure la plus importante, la plus significative, est celle du début du travail. Elle marque la rupture avec le temps de non-travail $\gg .^{30}$

Cette quantification va déboucher sur la monopolisation des représentations de la temporalité par le temps de travail, lequel devient le moteur même de la 
représentation socioculturelle de la temporalité qui caractérise la modernitési ${ }^{31}$ Toute une logique temporelle spécifique émerge à partir du temps de travail et de l'ensemble du système productif. Le temps devient de plus en plus abstrait - voire objectif - en ce sens qu'il perd sa contingence au profit d'une nécessité qui obéit à une logique productive dont l'horloge, la montre et le chronomètre deviennent des symboles de ce temps répétitif et mécanique ${ }^{32}$. À telle enseigne que cette temporalité objective et contraignante $^{33}$ fera dire à plusieurs qu'on est en face d'une véritable dictature du temps ${ }^{34}$.

\section{Productivité, productivisme et fétichisme de l'urgence}

9 La logique du temps objectif et abstrait, quantifié et quantifiable, va reposer très tôt dans la modernité sur une certaine idéologie du progrès ${ }^{35}$. Une idéologie fondée sur la quête d'un certain progrès social qui va de pair avec le contrôle de la nature, ici du temps, comme valeur dominante des sociétés modernes. La connaissance scientifique, les développements techniques et le contrôle du temps qui donnent l'impression d'une maitrise absolue de la temporalité vont constituer les dimensions fondamentales de cette idéologie du progrès, pour ainsi faire des individus, selon l'aphorisme bien connu, des maîtres et possesseurs de la nature. ${ }^{36}$ C'est l'idée de la mathématisation universelle conforme à la mathesis universalis développée par Descartes qui voit ses prolongements pénétrer toutes les formes de la vie sociale. Mathématisation, quantification et abstraction du temps vont devenir les composantes clés de ce contrôle du temps, de cette maîtrise du temps. Celle-ci se fait à travers l'invention du concept et de la mesure de productivité initialement posée par les économistes classiques tels que Smith, Ricardo, Say et d'autres, lesquels vont théoriser pour la première fois l'idée selon laquelle «le temps c'est de l'argent ", ce que Benjamin Franklin reprendra à son compte dans une formule bien connue aujourd'hui: "Time is money». Il s'agit là, on le comprendra, d'un enjeu majeur pour le capitalisme, en ce sens que la productivité va constituer l'indicateur par excellence de la croissance économique. C'est dans cette optique que la mesure de la productivité devient fondamentale, dans un dessein de prévision et d'anticipation du fonctionnement des grandes structures économiques. Or cette notion, classique et néoclassique ${ }^{37}$, de la productivité repose sur l'idée de la "performance» comme appréciation qualitative d'un processus de production quant à son rendement, son efficacité, sa rentabilité et son degré de compétitivité. Il s'agit donc d'un concept global qui permet de porter un jugement sur un processus de production de façon générale ${ }^{38}$.

10 C'est précisément cette idée de performance qui va poser la maîtrise toujours plus poussée du temps comme un enjeu majeur pour les sociétés occidentales modernes. Ce qui va conduire au développement sans précédent de la rationalité, on pourrait dire de l'hyperationalité, dans toutes les sphères d'activités sociales. L'objectif étant ici de contrôler toujours plus le temps, de lui accorder une finalité productiviste. Ce qui pour les penseurs classiques en économie va consister à trouver des stratégies d'augmentation de la performance au travail (force de travail) qui sera possible, d'une part, par une gestion rationnelle de la force de travail elle-même en milieu industriel et, d'autre part, par la reproduction de cette même force à l'extérieur des heures de travail (reproduction élargie). C'est ainsi que les idées de Taylor vont trouver plusieurs échos, toujours dans cette idée d'anticiper la productivité par l'imposition, dans les 
organisations industrielles, de critères de performances dont le but est d'assurer le meilleur rendement possible, comme en témoigne ce passage : «L'objet principale d'une organisation bien comprise doit être d'assurer à l'employeur et à l'employé la prospérité maxima $»{ }^{39} \mathrm{Ce}$ qui suppose ainsi une organisation rationnelle du travail, fondée sur une maîtrise absolue du temps. D'où la nécessité de minutieusement chronométrer les tâches et gestes de tous les travailleurs suivant des règles prévues par des spécialistes des ingénieurs du temps ${ }^{40}$ - dont les relevés devront être maintenus à l'écart des ouvriers dans des bureaux prévus à cette fin. Une séparation entre conception et exécution du produit du travail s'impose ainsi, toujours dans le but d'intensifier le travail au maximum par une division des différentes tâches et de celles-ci dans le temps ${ }^{41}$.

11 D'autre part, selon Taylor, cette organisation rationnelle du travail, qu'il va nommer organisation scientifique du travail, devait reposer sur un nouveau système de salaire, encourageant la production toujours plus élevée des ouvriers eux-mêmes. D'où l'importance de la vitesse, étroitement associée à la productivité et à la performance. C'est cette vitesse, ce désir d'aller toujours plus vite, de faire le plus de choses possible dans un temps de plus en plus court (productivité), qui va devenir précisément le fondement de la représentation dominante de la temporalité au XXe siècle et encore aujourd'hui ${ }^{42}$.

12 La vitesse a de tout temps été considérée dans son rapport à l'espace et au mouvement. Depuis la physique d'Aristote, élaborée dans le livre IV de Physique, la vitesse est considérée comme le mouvement entre une borne antérieure et une borne postérieure, lesquelles sont traversées par des instants qui sont quantifiés. Or, avec l'arrivée des TIC et la généralisation de celles-ci dans la plupart des organisations, la vitesse tend à se détacher de l'expérience subjective qui l'appréhende en rapport avec la distance parcourue entre deux points. Plusieurs dimensions importances des TIC permettent d'avancer ce propos. Premièrement, la possibilité de communiquer à distance en évitant les impératifs de l'espace nécessitant les déplacements. Deuxièmement et conséquemment, la disparition de l'espace comme lieu de déploiement du temps. Et troisièmement, la possibilité de traiter plusieurs informations simultanément, en même temps.

Bref, cette idéologie d'une maîtrise absolue du temps par une augmentation de la performance et de la vitesse aura plusieurs impacts chez les individus et dans les organisations, qui verront leurs rythmes se transformer suivant les rythmes objectifs et contraignants de la productivité industrielle. Ainsi, l'efficacité, la performance, l'efficience, la vitesse et la rapidité viendront façonner l'univers des représentations des individus qui seront désormais soumis à cette pression temporelle, découlant de cette « obsession de la vitesse et de la performance qui font de la vitesse une qualité en soi » ${ }^{43}$. C'est cette pression temporelle que plusieurs psychologues ont considéré comme source de l'émergence d'une certaine pathologie temporelle ${ }^{44}$, fondée sur le fétichisme ou ce culte de l'urgence généralisée $e^{45}$, qui s'imposera aux individus et qui exigera une appropriation individuelle de plus en plus grande du temps ${ }^{46}$. On ne s'étonnera donc pas de l'individualisme, par rapport au temps, qui en découle. Les jeunes verront dès leur jeune âge se mettre en place des structures (écoles, télévision éducative, apprentissages familiales, etc.) visant à créer un univers de représentations par lequel ils seront contraints d'accorder une importance centrale à la réussite individuelle et à la performance tout en développant, à l'inverse, un sentiment de culpabilité face à 
l'échec, face à l'oisivetée ${ }^{47}$. Tant et si bien qu'un nombre grandissant d'individus justifieront leur utilisation personnelle des TIC par une obsession avouée de la vitesse et de la rapidité, c'est-à-dire par une volonté de faire le plus d'activités possible dans un temps de plus en plus court ${ }^{48}$. C'est aussi dans ce contexte qu'on va voir apparaitre des "pathologies de l'attente », c'est-à-dire l'incapacité pour certains individus d'inscrire leurs actions dans un temps qui n'est pas présentifiée ${ }^{49}$, par conséquent de tout ramener à l'urgence quitte à abolir le temps même ${ }^{50}$. Il s'agit là d'un " désir de l'immédiat " ${ }^{51}$ où l'attente est vécue comme à priori "trop longue » et où par conséquent l'individu est toujours pressé5 ${ }^{52}$. C'est dans la représentation dominante qui prévaut actuellement que cette impatience chronique et pathologique trouve son origine, à partir du moment où les individus sont enfermés dans un certain moment présent. Ce qui implique le désir de rendre toutes ses tâches simultanées, de les poser dans un «moment actuel ». Dès lors, il n'y a plus de successions d'événements : tout se fait dans un certain maintenant. Le temps est dès lors posé dans son rapport à l'instant présent, à sa capacité de faire en sorte qu'un résultat se présente sans délais. Les individus se trouvent ainsi prisonniers dans un univers du « déjà ».

\section{L'organisation aujourd'hui : entre « dilatation spatiale » et « contraction temporelle »}

$\mathrm{Au}$ cours du XXe siècle, des transformations radicales du cadre spatio-temporel au sein des organisations se sont opérées. Nous sommes passés d'une époque d'adaptation longue et continue (temps long) au cours de laquelle la compétition entre les organisations s'exprimait sur une base locale (espace court), à une époque où les organisations entrent en compétition avec des concurrents à l'échelle mondiale (espace long) et doivent s'adapter rapidement aux évolutions du marché (temps court) ${ }^{53}$. Dans ce nouveau contexte, pour des raisons d'efficacité ${ }^{54}$, la maîtrise et le contrôle du temps et de l'espace deviennent des facteurs essentiels pour la réussite socioéconomique de l'organisation. Pour être en mesure de réagir vite, de conquérir des parts de marché et ainsi intégrer ce nouvel espace économique, les organisations sont amenées à fonctionner en réseaux ${ }^{55}$ et à développer des instruments spécifiques de gestion (par exemple, les progiciels) qui reconfigurent l'organisation du travail ${ }^{56}$, dans le sens d'un véritable reengineering ${ }^{57}$. Nombreux sont les chercheurs ${ }^{58}$ qui font le constat que les organisations sont entrées dans un mouvement de valorisation de l'urgence, vers une "idéologie du temps réel », recherchant la flexibilité du temps et du lieu de travail. Par exemple, le télétravail est « un des instruments de la flexibilisation et de l'injonction à agir dans l'urgence qui ont cours dans les entreprises $"^{59}$. Il se forge alors dans les organisations de nouvelles formes de temporalité, des nouvelles méthodes d'organisation par rapport au temps. La dimension temporelle est de plus en plus une variable d'action pour les organisations au sein desquelles se développe une véritable "ingénierie temporelle " ${ }^{60}$. Les individus travaillent dans un contexte de flux tendus ou de "juste à temps [qui] peut se résumer en une succession de zéros : zéro stock, zéro délais auquel on ajoutera zéro défaut " ${ }^{61}$. Par ailleurs, la variable temporelle fait partie de la culture de certaines entreprises qui développent leur stratégie de marketing sur leur capacité à satisfaire le client dans l'urgence (Feedex, photo minute, clé minute). Ainsi pour les employés, travailler dans l'urgence devient la norme et l'homo-urgentus doit arriver à gérer et maîtriser le temps. 


\section{Les TIC instruments soutenant « une idéologie de l'urgence »}

15 Loin de nous l'idée d'adopter une position déterministe, mais un constat est fait ${ }^{62}$ : les TIC soutiennent l'implantation d'une «idéologie de l'urgence» dans les organisations. Grossin ${ }^{63}$ souligne d'ailleurs que l'utilisation des TIC encourage " une flexibilité qui se répercute sur les modalités temporelles de l'organisation du temps de travail $»^{64}$. Ainsi, des choix organisationnels couplés à l'introduction des TIC peuvent être des outils mis au service d'une intensification de la cadence de travail et d'une lutte aux temps morts ${ }^{65}$. Les TIC " permettent la connexion, [et] leur usage est structuré par une tension permanente entre l'autonomie et le contrôle " ${ }^{66}$. Par ailleurs, comme nous l'avons souligné plus haut, le fait de pouvoir se connecter à distance et à tout moment modifie notre rapport du travail au temps ${ }^{67}$. Le temps de travail implique à la fois des temps de présence sur le lieu de travail, mais aussi des périodes de disponibilités plus larges, des tâches à effectuer à domicile, des rythmes plus flexibles. Les TIC permettent au travailleur d'être en lien direct avec son organisation (courrier électronique, téléphones portables) et favorisent une intrusion du travail dans la sphère privée ${ }^{68}$, opérant ainsi un véritable "brouillage des frontières $»^{69}$.

16 Vendramin et Valenduc ${ }^{70}$ suite à une série d'études de cas menées dans six pays européens font le constat que les TIC sont complices de l'intensification du travail et participent au renouveau des principes tayloriens ${ }^{71}$. Ce phénomène s'est aussi posé avec acuité dans les services publics, dont les services hospitaliers qui, comme l'a souligné Gadrey, constituaient une cible de choix de ce renouveau taylorien. « Compte tenu de leur poids dans l'activité et dans les dépenses, les services hospitaliers semblent d'excellents candidats pour la recherche de "gains de productivité», et la plupart des discours sur la maitrise des dépenses de santé font état de l'existence de "gisements de productivité» qui, dans l'état actuel des choses, resteraient inexploités $\gg .{ }^{72}$ De là, la nécessité, pour l'État ${ }^{73}$, de saisir les opportunités offertes par les TIC pour transformer radicalement l'organisation du travail médical suivant une logique productiviste tayloriste ${ }^{74}$.

17 Les TIC contribuent à prescrire la gestion des flux informationnels au sein de l'organisation, à opérer un contrôle sur la gestion du temps et transforment la nature du travail en augmentant la charge informationnelle. La prise en main du flux des échanges par des systèmes informatiques, le fractionnement des chaînes de travail, sont des traces de cette nouvelle taylorisation des activités tant industrielles que de services $^{75}$. Par exemple, les logiciels de Workflow recréent des chaînes de production virtuelle en réintroduisant le chronométrage et l'enchaînement séquentiel des tâches. "Le workflow (outil de développement de la productivité de groupe) met le travail sous haute surveillance, il contrôle les temps, les tâches, et produit une batterie d'indicateurs sur le travail de chacun qui met le salarié dans une situation de contrôle et de dépendance inédite ${ }^{76}$. De tels outils de travail impliquent une gestion centralisée de l'information et appliquent un principe de gestion à flux tendus aux flux informationnels. Toutes données et informations doivent être entrées dans le système régulièrement et la responsabilité incombe aux employés de les intégrer (encoder) correctement et au bon moment afin que d'autres opérateurs soient en mesure d'effectuer leur travail ${ }^{77}$. Par ailleurs, ces systèmes en produisant des indicateurs sur le travail de chacun sont l'expression d'une obsession de la mesure (par exemple, le temps acceptable pour répondre en ligne dans 
un service à la clientèle, le temps nécessaire pour traiter un dossier dans une compagnie d'assurance, etc.). Ces technologies appliquées à la gestion des activités de l'organisation contractent le temps en le décomposant plus finement. L'objectif est d'optimiser la gestion du temps, de tirer profit au maximum du temps qui passe, de lutter contre les temps morts ${ }^{78}$. «[Les] logiciels (PGI et workflow) ont pour objet de prescrire de manière très directive le contenu et l'enchaînement des tâches à effectuer, en éliminant toute porosité dans la circulation de l'information et dans le temps passé par les opérateurs à organiser cette circulation de l'information. Dans les systèmes taylorisés, le stress du flux remplace l'autorité du contremaître $»^{79}$. Autrement dit, les TIC ont influencé l'organisation du travail et elles sont complices de la mise en place de dispositifs qui accroissent la pression sur le temps, induisant ainsi, comme nous l'avons souligné plus haut, un changement radical du cadre spatio-temporel (réduire les délais de réaction, tout en élargissant l'espace de travail).

Des outils technologiques tels que l'intranet, le forum de discussion, le courrier électronique permettent aux travailleurs d'être connectés et d'être informés rapidement, tout en étant aussi des facteurs d'intensification et de «densification $d u$ travail $\aleph^{80}$. Saintive ${ }^{81}$ a mené une recherche au sein du groupe EDF (Électricité de France) afin d'étudier l'impact des TIC sur le travail et son organisation ${ }^{82}$. Elle constate notamment que la messagerie modifie l'organisation du travail en imposant une cadence et un rythme de déroulement des activités : raccourcissement des délais de circulation de l'information, flux et traitement croissants de l'information, communication avec divers interlocuteurs sur des sujets variés dans un temps réduit, parcellisation du travail devenu imprévisible et rendant difficile la gestion des activités programmées et des tâches annexes réalisées via la messagerie. Les individus sont donc dépendants d'un rythme imposé par l'outil dans un souci de maintenir la cadence.

La messagerie électronique ou les forums de discussion sont par ailleurs des outils permettant d'obtenir rapidement un avis, une information de collègues, mais ce sont aussi des dispositifs favorisant la circulation des injonctions multiples et parfois contradictoires pouvant ainsi entraver le fonctionnement de l'organisation (confusion dans les demandes, dans les informations diffusées qui peuvent impliquer des réponses aux clients mal adaptées) ${ }^{83}$. Nous avons pu constater que "l'individu qui communique via un forum de discussion doit se débrouiller avec divers outils technologiques à sa disposition. Il interprète comme il peut les messages qu'il reçoit et y répond du mieux qu'il peut. Autrement dit, il auto-organise sa réalité et l'irruption permanente de l'environnement et de l'autre l'oblige à une constante réorganisation $»^{84}$.

20 Par ailleurs, l'instantanéité relationnelle permise par l'usage de la messagerie électronique ou du forum favorise l'expression d'une nouvelle forme de proximité (de la proximité physique à la proximité virtuelle) générant chez les utilisateurs un besoin de répondre rapidement et de manière satisfaisante à toute demande. Nous ne pouvons pas faire abstraction du fait que les TIC présentent une double réalité. En effet, elles peuvent à la fois faciliter l'exécution du travail (dossier partagé, travail collaboratif, etc.) et engendrer une surcharge cognitive voire une confusion du fait d'injonctions multiples et paradoxales. L'utilisation intensive du courrier électronique oblige les travailleurs à faire face à une surcharge informationnelle ayant pour conséquence l'augmentation de la charge mentale et accentuant le risque de syndrome de débordement cognitif ${ }^{85}$ ou "Cognitive Overflow Syndrom" (COS). Au sein des organisations, on assiste à une production croissante d'informations (notes, mémos, 
flux de messages via le courrier électronique) et de nombreuses personnes se plaignent d'être "noyées" sous une masse imposante d'informations qu'elles jugent inutiles. Ainsi, les personnes se disent trop sollicitées et expriment leur manque de temps, les retards dans l'avancement de certaines tâches et ont le sentiment d'une perte de contrôle sur leur activité quotidienne. C'est d'ailleurs ce que nous avons déjà constaté dans le secteur de la santé à la suite du déploiement des TIC dans les organisations de soins et de l'obligation, dans certains cas, pour les professionnels de la santé, d'informatiser leurs pratiques quotidiennes ${ }^{86}$. Lahlou ${ }^{87}$ explique ce phénomène par le fait que les sujets naviguent entre de multiples "attracteurs cognitifs» (ensemble d'éléments matériels et immatériels qui participent à une activité et sont simultanément présents dans l'environnement de travail du sujet). Les TIC accroissent le syndrome de débordement cognitif (COS) parce qu'elles permettent une multiplication des attracteurs. Ainsi, un environnement de travail riche en attracteurs tend à provoquer du «papillonnage» et de la «proscratination»: les travailleurs s'épuisent à vouloir réaliser une série de tâches urgentes et n'arrivent plus à gérer leurs priorités. Ce qui se traduit, dans certains cas au sein d'une même organisation, par l'émergence de deux organisations complètement parallèles du travail, la première fondée sur les anciens modes de fonctionnement et la deuxième sur l'utilisation quotidienne des TIC ${ }^{88}$.

\section{TIC complices d'un jeu de confrontations : de la " chrono-compétition » à la « déconnexion »}

21 Une composante normative implicite du discours de l'urgence est que : «être occupé c'est bien ${ }^{89}$. Un travailleur efficace est censé vivre sous pression temporelle perpétuelle. Cependant, les individus ne réagissent pas tous de la même façon à la flexibilité du temps, à la pression du temps. Pour les uns, l'urgence sera vécue comme une contrainte (obligation d'agir vite) et pour les autres comme un moyen d'être valorisé (être reconnu pour sa maîtrise de l'urgence) et l'expression d'une forme de pouvoir. Ainsi, le rapport que les membres de l'organisation entretiennent avec l'urgence est sous-tendu par deux logiques qui peuvent au sein d'une organisation s'affronter: soit une logique de survie et une logique de pouvoir ${ }^{90}$. Ces logiques sont directement liées à des comportements spécifiques qui peuvent s'opposer et entrer en contradiction. Néanmoins, dans certaines organisations, des stratégies implicites de lutte contre la pression du temps s'érigent en véritables normes collectives. On voit par exemple émerger des conventions d'usage de la messagerie dans la manière de rédiger un message électronique (libeller avec précision les objets du message, rédiger des messages courts, cibler précisément les destinataires, etc.).

$\mathrm{Au}$ sein des organisations, de nouvelles pratiques communicationnelles se mettent en place et les individus ont tendance à valoriser le " geste instrumentalisé » ${ }^{91}$ plutôt que de l'acte de parole. Nous avons pu observer au cours d'une recherche sur l'usage du forum de discussion en situation de formation à distance que les usagers s'approprient les dispositifs techniques (courrier électronique ou forum de discussion) et vont souvent l'adapter à leurs propres besoins (besoin de collaboration), redéfinissant ainsi leur espace de communication ${ }^{92}$. Par exemple, les utilisateurs retiennent certaines fonctionnalités techniques intégrées à l'outil (comme les fonctions "citation", "commentaire» dans un forum ou les fonctions "répondre», "transférer» dans une 
messagerie) pour en abandonner d'autres, intégrant ainsi l'outil à leurs pratiques de communication. Certaines fonctionnalités seront donc "agies" et exerceront une action régulatrice sur les échanges, renforçant par exemple la dimension interpersonnelle d'un échange à distance et soutenant par la même occasion l'accomplissement d'un engagement du sujet dans l'interaction. On voit ainsi apparaître des formes de "ritualisation sociale » régulant les échanges à distance ${ }^{93}$. Ainsi, au cours d'une activité collaborative certains individus vont faire des efforts manifestes pour maintenir leur engagement envers leurs collaborateurs et consolider la cohésion et la productivité d'un groupe de travail, alors que d'autres vont adopter un comportement d'attente signe de leur non engagement. Par conséquent, nous pouvons dire que les TIC ne se limitent pas à nous renseigner sur les rapports entre les individus, mais elles agissent sur ces rapports et, dans une certaine mesure, les constituent. Ces dispositifs technologiques acquièrent un statut d'agent social de par leur capacité à " agir » et à " faire agir » en modifiant les inter-relations ${ }^{94}$.

La nouvelle économie nous a fait entrer dans l'ère de la compétition basée sur le temps. De nouvelles pratiques de management ont vu le jour puisque se développe dans les organisations une nouvelle forme de "nomadisme électronique $»^{95}$ permettant aux individus d'entrer dans le jeu de la "chrono-compétition ${ }^{96}$, mais aussi d'occuper plusieurs emplois au cours de leur vie ${ }^{97} \mathrm{du}$ fait de leur capacité à s'adapter rapidement au changement compte tenu de leur maîtrise des TIC et du temps. Donner des réponses à temps et avant tout le monde, supprimer les temps morts pour gagner en réactivité et en efficacité est devenu pour certains acteurs la possibilité de se valoriser au travail et d'y trouver une certaine forme de satisfaction. La technologie mobile (téléphone mobile, ordinateur portable, agenda électronique), la mise en réseau via Intranet des ressources de l'organisation permettent aux individus d'accroître la flexibilité de leur temps et lieu de travail ${ }^{98}$. Isaac ${ }^{99}$ étudie l'émergence d'une forme de "nomadisme $d u$ manager $»^{100}$ contribuant à doter le management de caractéristique d'ubiquité et d'entrer dans le jeu de la "chrono-compétition ${ }^{101}$. Cependant, ce nomadisme présent dans de nombreuses organisations soulève une interrogation: "la disponibilité permanente génère une culture de l'instantanéité qui peut influencer la prise de décision. La confusion entre l'urgence et l'importance d'une tâche à effectuer devient souvent très floue pour beaucoup de managers $»^{102}$. A contrario, le rythme imposé par l'outil informatique (messagerie, progiciels et autres) génère un stress chez certaines personnes par crainte de la déconnexion et du risque de laisser échapper des informations, d'être submergé d'informations, de mal gérer son temps ${ }^{103}$. Face à ce sentiment d'oppression, certains individus vont développer des stratégies individuelles de freinage pour retrouver un peu de pouvoir sur soi, sur l'organisation de leur temps et de leur activité.

\section{Conclusion}

Dans la plupart des analyses se rapportant à l'organisation, la dimension temporelle est quelque peu occultée ou insuffisamment prise en compte. La réflexion menée dans ce texte nous a permis de souligner le fait que le temps est une contrainte qui s'impose indéniablement aux organisations et à leurs membres. De nouveaux modes de gestion s'appuyant sur les TIC permettent aux employés « d'être à la fois plus autonomes et plus contrôlés, [et] de réagir plus vite aux multiples sollicitations de l'activité productive " ${ }^{104}$. On voit se mettre en place un «nouveau taylorisme à distance $»^{105}$ confrontant alors les sujets à 
une double injonction (injonction paradoxale) en les soumettant à la fois à une exigence d'autonomie (associée à l'incitation à travailler en équipe, à collaborer, à prendre des décisions efficaces et rapides) et une exigence de contrôle. Cette logique de l'urgence qui gouverne aujourd'hui les organisations tend à imposer une double contrainte aux individus, laquelle contribue à la mise en place d'un système de communication paradoxale au sein de l'organisation.

\section{BIBLIOGRAPHIE}

Attali J. (1982). Histoire du temps, Paris, Fayard.

Aubert, N. (1998). Le sens de l'urgence, Sciences de la société, 44, p. 29-42.

Aubert, N. (2003a). Le culte de l'urgence, la société malade du temps. Paris : Flammarion.

Aubert N. (2003b). «Urgence et instantanéité : les nouveaux pièges du temps », dans Modernité : la nouvelle carte du temps (sous la direction de François Asher et Francis Godard), Éditions de l'Aube Le Moulin du Château, p. 169-185.

Aubert, N. (2004). Les entreprises face à l'urgence : Y-a-t-il encore place pour l'anticipation?, Information sur les sciences sociales, vol. 43(3), p. 309-402.

Barreau H. (1996). Le temps, Paris, PUF.

Bayart D. (1998). Temps et organisation : vers une ingénierie temporelle, Revue Française des Affaires Sociales, vol. 52, n 3, juillet-septembre 1998, p. 19-34.

Bergson H. (1948). Essai sur les données immédiates de la conscience, Paris, PUF.

Bonneville, L. (2005a). L'incidence de l'utilisation quotidienne d'un système de communication informatisé sur la pratique infirmière : le cas du système d'information géronto-gériatrique (SIGG) dans la région des Bois-Francs (Québec), Rapport de recherche, Ottawa, Université d'Ottawa, 2005 (à paraître, texte ronéotypé).

Bonneville, L. (2005b). « L'informatisation comme outil de contrôle et de surveillance de la productivité des organisations de soins et du travail médical au Québec », Revue Terminal Technologies de l'information, culture et société, no. 92, hiver 2004-2005, p. 173-185.

Bonneville, L. (2005c). « La transformation des organisations de soins et du travail médical par le recours à l'informatisation au Québec : une analyse critique ", Revue Communication et Organisation, no. 26, printemps 2005, p. 205-225.

Bonneville, L. (2003a). « La mise en place du virage ambulatoire informatisé comme solution à la crise de productivité du système sociosanitaire au Québec (1975 à 2000) », Thèse de doctorat en sociologie. Montréal : Université du Québec à Montréal.

Bonneville L. (2003b). « Informatisation sociale et représentation de la temporalité », dans JeanPierre Dupuis (sous la direction), Des sociétés en mutation, Montréal, Nota Bene, p. 77-92. 
Bonneville L. (2002). « La temporalité du réseau Internet est-elle encore moderne? », dans Jauréguiberry Francis et Serge Proulx (sous la direction), Internet, nouvel espace citoyen ?, Paris : L'Harmattan, p. 205-222.

Brousseau, E., Rallet, A. (1997). «Le rôle des technologies de l'information et de la communication dans les changements organisationnels ", dans Guilham, Bernard (sous la direction), Économie de la connaissance et organisations. Entreprises, territoires, réseaux. Paris, Montréal : L'Harmattan, p. 286-309.

Bühler, N., Ettighoffer, D. (1995). « L'homme polyactif », dans Gérard Blanc (sous la direction), Le travail au XXIe siècle : mutations de l'économie et de la société à l'ère des autoroutes de l'information, Paris, Dunod, p. 203-218.

Caccomo, J.L (1996). Les défis économiques de l'information : la numérisation, Paris, L’Harmattan.

Caillois R. (1976). « La Fête », Euvres, Paris, Gallimard.

Cassirer E. (1975). Essai sur l'homme, Paris, Éditions de Minuit.

Castells, M. (1998). L’ère de l'information, vol. 1 : La société en réseau, Paris, Fayard.

Castells, M. (2001). La galaxie Internet, Fayard, Paris.

Condorcet Marie Jean Antoine Caritat marquis de. (1970). Esquisse d'un tableau historique des progrès de l'esprit humain, Paris, Vrin (édition originale en 1798).

Coriat B. (1990). L'atelier et le robot, Paris, Bourgois.

Descartes R. (1962). Discours de la méthode, Paris, Presses de l'imprimerie Bussière, (édition originale en 1637).

Durkheim É. (1986). Les Règles de la méthode sociologique, Paris, PUF (édition originale en 1895).

Ettighoffer, D. (1995). « Networkers, les nomades électroniques», dans Gérard Blanc (sous la direction), Le travail au XXI ${ }^{e}$ siècle : mutations de l'économie et de la société à l'ère des autoroutes de l'information, Paris, Dunod, p. 219-236.

Flichy, P. (2004). « L'individualisme connecté entre la technique numérique et la société », Réseaux, $\mathrm{n}^{\circ} 124$, p. 38.

Gadrey, J. (1992). L'économie des services, Paris, Éditions La Découverte.

Grosjean, S. (2005). « Communautés virtuelles et communication médiatisée par ordinateur : une analyse des formes de « ritualisation sociale ». Revue d'Interaction Homme-Machine, RIHM, Paris, p. 107-129.

Grosjean, S. (2004). « Analyse d'une forme de communication instrumentée lors d'activité collaborative de conception ». Revue Les Enjeux de l'information et de la communication, Université Stendhal, Grenoble (France). Disponible en ligne : http://www.u-grenoble3.fr/les_enjeux/2004/ Grojean/index.html

Grosjean, S. (2004). «L'apprentissage collaboratif à distance : du scénario pédagogique à la dynamique interactionnelle ». Actes du colloque Technologies de l'Information et de la Connaissance dans l'Enseignement supérieur et l'industrie, TICE'2004, Université de Technologie (UTC), Compiègne (France), 28-30 octobre 2004, p. 229-236.

Grossin, W. (1998). « Les temps de travail », In Coster, M., Pichault, F. (Eds), Traité de sociologie du travail, De Boeck, Bruxelle, 139-155. 
Grossin, W. (1969). Le travail et le temps : horaires, durées, rythmes : une enquête dans la construction mécanique et électrique de la région parisienne, Paris, Anthropos, p. 207-209.

Hammer, M., Champy, J. (1993). Le reengineering, Paris, Dunod.

Isaac, H. (2004). « Technologie mobile et management : émergence d'un manager nomade?». Disponible en ligne : http://www.aim2004.int-evry.fr/pdf/Aim04_Isaac.pdf

Jauréguiberry, F. (2003). « La mise en place des télécommunications mobiles au sein des entreprises comme nouvelles formes d'exploitation ", Communication aux IXes journées de Sociologie du travail, Novembre 2003.

Jaureguiberry, F. (2003). Les branchés du portable, Paris, PUF.

Jauréguiberry, F. (1998), « Télécommunications et généralisation de l'urgence», Sciences de la société, no. 44, 1998, p. 83-96.

Lahlou, S., «Attracteurs cognitifs et travail de bureau », Intellectica, 2000/1, 30, pp. 75-113.

Lahlou, S., Lenay, C., Gueniffey, Y., Zacklad, M. (1997). « Le COS, tel que défini par L'ARC », In Compte-rendu de la $152^{\text {ème }}$ réunion du CA de l'ARC, Bulletin de l'Association pour la Recherche Cognitive, $n^{\circ}$ 42, novembre 1997, p. 39.

Lamarche F. et Doré M. (1980) «L'organisation du travail et les travailleurs ", dans La crise et les travailleurs, Montréal, co-édition Faculté de l'éducation permanente de l'Université de Montréal et Centrale de l'enseignement du Québec, p. 83-91.

Langlois G., Villemure G. (1992). Histoire de la civilisation occidentale, Montréal, Éditions Beauchemin.

Lechat, N., Delaunay, J-C. (2003). Les centres d'appel : un secteur en clair-obscur. L'Harmattan : Paris.

Lemaire, L. (2003). Systèmes de gestion intégrés : des technologies à risques. Editions Liaisons, collection Entreprises \& Carrières : Paris.

Lévi-Srauss C. (1969). Anthropologie structurale, Paris, Plon.

Mayère, A. (1999). « Les effets de l'introduction d'outils d'information et de communication mobile dans l'entreprise », Revue Française de Gestion, Mars-Avril, p. 88-99.

Mercure D. (1989). «Les temporalités vécues dans les sociétés industrielles », dans Temps et société (sous la direction de Gilles Pronovost et Daniel Mercure), Québec, Institut de recherche sur la société et la culture.

Metzger, J-L., Cléach, O. (2004). « Le télétravail des cadres : entre suractivité et apprentissage de nouvelles temporalités », Sociologie du travail, vol. 46, n 4, p. 431-574.

Mircea, E. (1961). Le mythe de l'éternel retour : archétypes et répétition, Paris, Gallimard.

Ouvrage collectif. (1975). Les cultures et le temps, ouvrage collectif, Payot/UNESCO, Paris.

Pronovost G. (1996). Sociologie du temps, Bruxelles, De Boeack-Wesmael.

Pronovost G. et Mercure D. (1989). Temps et société, Québec, Institut Québécois de Recherche sur la Culture.

Pucelle J. (1967). Le temps, Paris, PUF.

Reinberg A. (1979). L'Homme malade du temps, Paris, Stock. 
Saintive, B. (2000). Trois formes différenciées d'usage de la messagerie électronique au sein d'une organisation, Réseaux, n 104, p. 119-136.

Saintive, B. (2002). Rôle de la messagerie électronique sur la communication et ses incidences sur l'organisation du travail et les utilisateurs, Acte du 35ème congrès de la Société d'Ergonomie de Langue Française, Communication et travail, Toulouse. Disponible en ligne : http://www.ergonomieself.org/actes/congres2000.html

Sue R. (1994). Temps et ordre social, Paris, PUF, 1994.

Smail, A.-E.-H. (1989). L'entreprise face à la mutation technologique, Paris, Éditions d'Organisation. Smith, A. (1937). An Inquiry to the Nature and Causes of the Wealth of Nations, New York, The Modern Library (édition originale en 1776) p. 3-11.

Stalk, G., Hout, T.M. (1990). Competing against time: how time-based competition is reshaping global markets, Free Press: New-York.

Taylor F.W. (1911). Principes d'organisation scientifique des usines, Paris, Dunod.

Torres, O. (2000). « L'entrepreneuriat face à la globalisation », dans Verstraete T. (ed.), Histoire d'entreprendre, Editions Management \& Société (EMS) : Paris.

Tremblay G. (1989). « Les mass médias, instruments de gestion du temps », dans Temps et société (sous la direction de Gilles Pronovost et Daniel Mercure), Québec, Institut de recherche sur la société et la culture, p. 127 et suivantes.

Usunier, J.C. (1995). Une critique de la fonctionnalité de l'urgence, Temporalistes, n² 29, p. 5-10.

Valenduc, G. (2005). La technologie, un jeu de société. Au-delà du déterminisme technologique et du constructivisme social. Collection Sciences et Enjeux, Academio Bruylant, Louvain.

Vendramin, P \& Valenduc, G. (2002). Technologies de l'information et de la communication, emploi et qualité de travail, Ministère de l'Emploi et du travail, Bruxelles.

Vinet, A., Bourbonnais R., Brisson C. (2003). « Travail et santé mentale. Une relation qui se détériore ", Dans Santé mentale et travail. L'urgence de penser autrement l'organisation (sous la direction de Jean-Pierre Brun, Christian Blais, Sylvie Montreuil et Alain Vinet), Sainte-Foy, PUL.

Zarifian P. (2001). Temps et modernité. Le temps comme enjeu du monde moderne, Paris, L'Harmattan.

\section{NOTES}

1. Bonneville, L. (2003a). « La mise en place du virage ambulatoire informatisé comme solution à la crise de productivité du système sociosanitaire au Québec (1975 à 2000)», Thèse de doctorat en sociologie. Montréal : Université du Québec à Montréal, chapitre 1.

2. Caccomo, J.L (1996). Les défis économiques de l'information: la numérisation, Paris, L'Harmattan, p. 11-12.

3. Aubert, N. (2003). Le culte de l'urgence, la société malade du temps. Paris : Flammarion.

4. Aubert N. (2003). "Urgence et instantanéité : les nouveaux pièges du temps», dans Modernité: la nouvelle carte du temps (sous la direction de François Asher et Francis Godard), Éditions de l'Aube - Le Moulin du Château, p. 169-185.

5. Stalk, G., Hout, T.M. (1990) Competing against time: how time-based competition is reshaping global markets. Free Press : New-York.

6. Aubert N. (2003a). Op. cit.; et Aubert, N. (2004). Les entreprises face à l'urgence : Y-a-t-il encore place pour l'anticipation?, Information sur les sciences sociales, vol. 43(3), p. 309-402. 
7. Pronovost G. (1996). Sociologie du temps, Bruxelles, De Boeack-Wesmael ; et Sue R. (1994). Temps et ordre social, Paris, PUF, 1994.

8. Durkheim É. (1986). Les Règles de la méthode sociologique, Paris, PUF (édition originale en 1895).

9. Sue R., (1994). Op. cit., p. 140.

10. Cassirer E. (1975). Essai sur l'homme, Paris, Éditions de Minuit.

11. Pronovost G. (1996). Op. cit. ; et Sue R. (1994). Op. cit..

12. Pronovost G. et Mercure D. (1989). Temps et société, Québec, Institut Québécois de Recherche sur la Culture, p. 10.

13. Ouvrage collectif, (1975). Les cultures et le temps, ouvrage collectif, Payot/UNESCO, Paris.

14. Tremblay G. (1989). "Les mass médias, instruments de gestion du temps ", dans Temps et société (sous la direction de Gilles Pronovost et Daniel Mercure), Québec, Institut de recherche sur la société et la culture, p. 127 et suivantes.

15. Attali J. (1982). Histoire du temps, Paris, A. Fayard.

16. Pronovost G. (1996). Op. cit., chapitre 3.

17. Barreau H. (1996). Le temps, Paris, PUF, p. 22 et suivantes ; et Pucelle J. (1967). Le temps, Paris, PUF, chapitre premier.

18. Bergson H. (1948). Essai sur les données immédiates de la conscience, Paris, PUF.

19. Barreau H. (1996). Op. cit., chapitre premier ; et Pucelle J. (1967). Op. cit., chapitre premier.

20. Barreau H. (1996). Ibid., chapitre premier ; et Pucelle J. (1967). Ibid., chapitre premier.

21. Caillois R. (1976). « La Fête», CEuvres, Paris, Gallimard ; et Mircea, E. (1961). Le mythe de l'éternel retour : archétypes et répétition, Paris, Gallimard.

22. Lévi-Srauss C. (1969). Anthropologie structurale, Paris, Plon.; et Mircea E., Ibid..

23. Caillois R. (1976). Op. cit..

24. Sue R. (1994). Op. cit., p. 33-36.

25. Langlois G. et Villemure G. (1992). Histoire de la civilisation occidentale, Montréal, Éditions Beauchemin.

26. Mercure D. (1989). «Les temporalités vécues dans les sociétés industrielles», dans Temps et société (sous la direction de Gilles Pronovost et Daniel Mercure), Québec, Institut de recherche sur la société et la culture, p. 22.

27. Condorcet Marie Jean Antoine Caritat marquis de, (1970). Esquisse d'un tableau historique des progrès de l'esprit humain, Paris, Vrin, (édition originale en 1798).

28. Pronovost G. (1996). Op. cit. ; et Sue R.. (1994). Op. cit..

29. Attali J. (1982). Op. cit., p. 190.

30. Grossin W. (1969). Le travail et le temps: horaires, durées, rythmes : une enquête dans la construction mécanique et électrique de la région parisienne, Paris, Anthropos, p. 207-209.

31. Sue R. (1994). Op. cit..

32. Attali J., Op. cit., et Coriat B. (1990). L'atelier et le robot, Paris, Bourgois.

33. Marx affirmera à ce sujet dans Misère de la philosophie que « le temps est tout, l'homme n'est plus rien ; il est tout au plus la carcasse du temps», cité dans Sue R., Op. cit., p. 74.

34. Zarifian P. (2001). Temps et modernité. Le temps comme enjeu du monde moderne, Paris, L'Harmattan.

35. Mercure D. (1989). Op. cit., p. 25.

36. Descartes R. (1962). Discours de la méthode, Paris, Presses de l'imprimerie Bussière, (édition originale en 1637).

37. Bonneville L. (2003). Op. cit., chapitre 1.

38. Ibid., chapitre 1.

39. Taylor F. W. (1911). Principes d'organisation scientifique des usines, Paris, Dunod, p. 31

40. Coriat B. (1990). Op. cit.. 
41. Voir, à ce propos, sur la division du travail, les explications fournies par Smith dans An Inquiry to the Nature and Causes of the Wealth of Nations (New York, The Modern Library, 1937 - édition originale en 1776 - p. 3-11 notamment).

42. Cette quête de vitesse va prolonger ce désir anthropologique de maîtriser le temps, de l'isoler, en l'objectivant par des instruments de mesure de plus en plus précis. Ainsi, en 1800 on mesurait le centième de seconde, puis vers 1850 la milliseconde. Le XXe siècle a été pour ainsi dire le siècle du début des grandes abstractions en ce qui a trait à la mesure du temps. En 1950 on mesurait la microseconde, en 1965 la nanoseconde, en 1970 la picoseconde, en 1990 la femtoseconde. On s'attend pour le prochain siècle à mesurer l'attoseconde. La conception de la vitesse dans le cadre de ces grands travaux de mesure du temps prend un tout autre sens par l'incapacité de l'être humain à percevoir, de lui-même, les instants séparant le point $\mathrm{A}$ du point $\mathrm{B}$.

43. Sue R., (1994). Op. cit., p. 11.

44. Reinberg A. (1979). L'Homme malade du temps, Paris, Stock.

45. Aubert N. (2003a). Op. cit. ; et Aubert N. (2003b).

46. Bonneville L. (2003). "Informatisation sociale et représentation de la temporalité ", dans Jean-Pierre Dupuis (sous la direction), Des sociétés en mutation, Montréal, Nota Bene, p. 77-92.

47. Bonneville L. (2002). «La temporalité du réseau Internet est-elle encore moderne? », dans Jauréguiberry Francis et Serge Proulx (sous la direction), Internet, nouvel espace citoyen?, Paris : L'Harmattan, p. 205-222.

48. Ibid.

49. Bonneville L. (2002). Op. cit.

50. Aubert N. (2003a). Op. cit., chapitre 5 ; et Aubert N. (1998). « Le sens de l'urgence», Sciences de la société, no. 44, p. 29-41.

51. Jauréguiberry F. (2003). Les branchés du portable, Paris, PUF, chapitre 2 notamment.

52. Jauréguiberry F. (2003). "L'homme branché, mobile et pressé ", dans Modernité: la nouvelle carte du temps (sous la direction de François Asher et Francis Godard), Op. cit., p. 155-168.

53. Torres O. (2000). "L'entrepreneuriat face à la globalisation ", dans Histoire d'entreprendre, (sous la direction de dans Verstraete Thierry), Éditions Management \& Société (EMS), Paris.

54. Brousseau É. et Rallet A. (1997). «Le rôle des technologies de l'information et de la communication dans les changements organisationnels ", dans Guilham, Bernard (sous la direction), Économie de la connaissance et organisations. Entreprises, territoires, réseaux, Paris, Montréal, L'Harmattan, p. 286-309.

55. Castells M. (1998) L'Ère de l'information, vol. 1 : La société en réseau, Fayard, Paris; et Castells M. (2001). La galaxie Internet, Fayard, Paris.

56. Lemaire L. (2003). Systèmes de gestion intégrés: des technologies à risques. Éditions Liaisons, collection Entreprise\&Carrières, Paris.

57. Le reengineering, est « une remise en cause fondamentale et une redéfinition radicale des processus opérationnels pour obtenir des gains spectaculaires dans les performances critiques que constituent aujourd'hui les coûts, la qualité, le service et la rapidité » (Hammer M. et Champy J. (1993). Le reengineering, Paris, Dunod, p. 42).

58. Aubert, N. (2004). Op. cit.; Valenduc, G. (2005). La technologie, un jeu de société. Au-delà du déterminisme technologique et $d u$ constructivisme social. Collection Sciences et Enjeux, Academio Bruylant: Louvain. et Mayère, A. (1999). Les effets de l'introduction d'outils d'information et de communication mobile dans l'entreprise, Revue Française de Gestion, Mars-Avril, p. 88-99.

59. Metzger J.-L. et Cléach O. (2004) « Le télétravail des cadres : entre suractivité et apprentissage de nouvelles temporalités ", Sociologie du travail, vol. 46, n 4, p. 431-574.

60. Bayart D. (1998). "Temps et organisation : vers une ingénierie temporelle », Revue Française des Affaires Sociales, vol. 52, $n^{\circ}$ 3, p. 19-34.

61. Vendramin P.et Valenduc G. (2002). Technologies de l'information et de la communication, emploi et qualité de travail, Ministère de l'Emploi et du travail, Bruxelles. 
62. Valenduc, G. (2005). Op. cit.; Saintive, B. (2000). «Trois formes différenciées d'usage de la messagerie électronique au sein d'une organisation ", Réseaux, n 104, p. 119-136.; (2002). « Rôle de la messagerie électronique sur la communication et ses incidences sur l'organisation du travail et les utilisateurs", Acte du 35ème congrès de la Société d'Ergonomie de Langue Française, Communication et travail, Toulouse. Disponible en ligne: http://www.ergonomieself.org/actes/congres2000.html ; Metzger, J-L., Cléach, O. (2004). Op. cit.. ; et Grossin, W. (1998). «Les temps de travail », In Coster, M., Pichault, F. (Eds), Traité de sociologie du travail, De Boeck, Bruxelle, 139-155.

63. Grossin, W. (1998). Op. cit..

64. Ibid.

65. Jauréguiberry F. (2003). Op. cit. ; et Bonneville L. (2005). «L'informatisation comme outil de contrôle et de surveillance de la productivité des organisations de soins et du travail médical au Québec ", Revue Terminal - Technologies de l'information, culture et société, N 92, hiver 2004-2005, p. $173-185$.

66. Flichy P. (2004). "L'individualisme connecté entre la technique numérique et la société », Réseaux, $\mathrm{n}^{\circ} 124$, p. 38.

67. Grossin W. (1969). Op. cit..

68. Soulignons, à ce titre, qu'une récente enquête a démontré que $30 \%$ des travailleurs canadiens (3 sur 10) apportent du travail à leur domicile (Vinet Alain, Bourbonnais Renée et Brisson Chantal. «Travail et santé mentale. Une relation qui se détériore», dans Santé mentale et travail. L'urgence de penser autrement l'organisation (sous la direction de Jean-Pierre Brun, Christian Blais, Sylvie Montreuil et Alain Vinet), Sainte-Foy, PUL, 2003, p. 13).

69. Flichy P. (2004) p. 18-51.

70. Vendramin, P \& Valenduc, G. (2002). Op. cit..

71. Ce qui, d'ailleurs, avait déjà été anticipé, en 1980, à la suite de l'introduction de l'informatique dans l'organisation du travail. Voir à ce propos F. Lamarche et M. Doré, «L'organisation du travail et les travailleurs", dans La crise et les travailleurs, Montréal, co-édition Faculté de l'éducation permanente de l'Université de Montréal et Centrale de l'enseignement du Québec, 1980, p. 83-91.

72. Gadrey J. (1992). L'économie des services, Paris, Éditions La Découverte, p. 77.

73. Bonneville L. (2003), op. cit..

74. Bonneville L. (2005), « La transformation des organisations de soins et du travail médical par le recours à l'informatisation au Québec: une analyse critique », Revue Communication et Organisation, no. 26, printemps 2005, p. 205-225 ; et Bonneville L. (2005b). art. cit..

75. Lechat N.t Delaunay J.C. (2003). Les centres d'appel: un secteur en clair-obscur, L'Harmattan: Paris.

76. Vendramin P.et Valenduc G. Op. cit..

77. Bonneville L. (2003), op. cit.

78. Ibid.

79. Vendramin P. et Valenduc G. (2002), op. cit.

80. Saintive B. (2000), art. cit..

81. Saintive, B. (2002), op. cit.

82. Ibid.

83. Flichy P, art. cit..

84. Grosjean S. (2005). «Communautés virtuelles et communication médiatisée par ordinateur: une analyse des formes de "ritualisation sociale", Revue d'Interaction Homme-Machine, RIHM, Paris, p. 107-129.

85. Lahlou S., Lenay C., Gueniffey Y. et Zacklad M. (1997). "Le COS, tel que défini par L'ARC », dans Compte-rendu de la $152^{\text {ème }}$ réunion du CA de l'ARC, Bulletin de l'Association pour la Recherche Cognitive, n 42, novembre 1997, p. 39. 
86. Bonneville L. (2003) Op. cit., chapitres 5 et 6.

87. Lahlou S. (2001). "Attracteurs cognitifs et travail de bureau», Intellectica, 2000/1, 30, pp. 75-113.

88. Bonneville L. (2005). L'incidence de l'utilisation quotidienne d'un système de communication informatisé sur la pratique infirmière: le cas du système d'information géronto-gériatrique (SIGG) dans la région des Bois-Francs (Québec), Rapport de recherche, Ottawa, Université d'Ottawa. (à paraître, texte ronéotypé).

89. Usunier J.-C. (1995). «Une critique de la fonctionnalité de l'urgence », Temporalistes, $\mathrm{n}^{\circ} 29$, 1995, p. 5-10.

90. Aubert N. (2003a) Op. cit. ; et Aubert N. (1998). art. cit..

91. Ces gestes peuvent être associés à des conduites non verbales propres aux échanges médiatisés et distants. Nous faisons allusion à la mobilisation par les sujets de fonctions techniques intégrées au dispositif de communication à distance (forum ou messagerie) qui permettent différents types d'action (citer, commenter, répondre, écrire, etc.). Pour des détails voir Grosjean S. (2004). «L'apprentissage collaboratif à distance : du scénario pédagogique à la dynamique interactionnelle ». Actes du colloque Technologies de l'Information et de la Connaissance dans l'Enseignement supérieur et l'industrie, TICE'2004, Université de Technologie (UTC), Compiègne (France), 28-30 octobre 2004, p. 229-236.

92. Grosjean S. (2005). art. cit..

93. Ibid.

94. Grosjean S. (2004). "Analyse d'une forme de communication instrumentée lors d'activité collaborative de conception ». Revue Les Enjeux de l'information et de la communication, Université Stendhal, Grenoble (France), disponible en ligne : http://www.u-grenoble3.fr/les_enjeux/2004/ Grojean/index.html

95. Ettighoffer D. (1995). « Networkers, les nomades électroniques », dans Gérard Blanc (sous la direction), Le travail au XXIe siècle: mutations de l'économie et de la société à l'ère des autoroutes de l'information, Paris, Dunod, p. 219-236.

96. Stalk G. et Hout T. M., (1990), op. cit.

97. Bühler N. et Ettighoffer D. (1995). "L'homme polyactif», dans Le travail au XXIe siècle: mutations de l'économie et de la société à l'ère des autoroutes de l'information, (sous la direction de Gérard Blanc), Paris, Dunod, p. 203-218.

98. Jauréguiberry F. (2003) op. cit.

99. Isaac, H. (2004). « Technologie mobile et management : émergence d'un manager nomade?». Disponible en ligne : http://www.aim2004.int-evry.fr/pdf/Aim04_Isaac.pdf

100. Isaac H. (2004), art. cit..

101. Stalk, G., Hout, T.M. (1990), op. cit.

102. Isaac $H,(2004)$, art. cit..

103. Saintive B. (2002), art. cit.

104. Flichy P. (2004),,art. cit..

105. Jaureguiberry F. (2003), op. cit. 


\section{RÉSUMÉS}

Les organisations contemporaines valorisent l'action et l'urgence. Par conséquent, la maîtrise et le contrôle du temps deviennent des enjeux fondamentaux de survie pour les organisations, contraintes à s'adapter à ce nouveau rythme socio-culturel. De nouveaux modes de gestion s'appuyant sur les TIC permettent aux employés d'être à la fois plus autonomes et plus contrôlés. Une forme de "taylorisme à distance » s'installe. Cependant, les individus ne réagissent pas tous de la même façon à la flexibilité du temps, à la pression du temps. En effet, certains individus entrent dans le jeu de la "chrono-compétition" alors que d'autres tentent de résister aux sollicitations multiples et à la pression du temps.

Contemporary organisations develops the action and the urgency. Consequently, the control of time become an important stake for organisations who wants to survive, in the goal of maintain their adaptation to this new socio-cultural rhythm. New forms of management being based on the ICT make it possible to the employees at the same time more autonomous and to be controlled. Today, a form of "distance Taylorism» exists. However, the employees react differently to the flexibility and pressure of time. Indeed, some peoples play the game of the « time-based competition », but others try to resist the pressure of time.

\section{INDEX}

Mots-clés : organisations, urgence, temporalité, vitesse, productivisme 